\title{
Analysis of intestinal microbial communities of cerebral infarction and ischemia patients based on high throughput sequencing technology and glucose and lipid metabolism
}

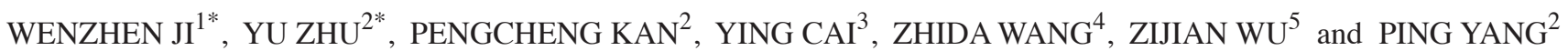 \\ Departments of ${ }^{1}$ Neurology and ${ }^{2}$ Clinical Laboratory; ${ }^{3}$ Tianjin Neurosurgery Institute, Tianjin Huanhu Hospital, \\ Tianjin Key Laboratory of Cerebral Vessels and Neural Degeneration, Tianjin 300350; ${ }^{4}$ Department of Renal Diseases, \\ Tianjin Medical University Metabolic Diseases Hospital, Tianjin 300070; ${ }^{5}$ Tianjin Key Laboratory of Food Biotechnology, \\ College of Biotechnology and Food Science, Tianjin University of Commerce, Tianjin 300134, P.R. China
}

Received October 21, 2016; Accepted June 13, 2017

DOI: $10.3892 / \mathrm{mmr} .2017 .7227$

\begin{abstract}
Currently, cerebral infarction (CI) is the leading cause of disability and the second leading cause of mortality in China, seriously affecting patient quality of life. Ischemia (IS) is considered to be the early stage of CI. The present study aims to investigate the variation of intestinal microbial communities in patients with CI and IS using high throughput sequencing technology, and then analyze the results to identify a novel potential pathogenic mechanism of CI and IS. In total, 8 patients with CI, 2 patients with IS and 10 healthy volunteers as a control were selected. Throughput sequencing technology was used to analyze the character and microbial population of the gut. The abundance of Escherichia, Bacteroides, Megamonas, Parabacteroides, Akkermansia, Prevotella, Faecalibacterium, Dialister, Bifidobacterium and Ruminococcus was the significant difference in the intestinal microbial communities of the $\mathrm{CI}$ and IS patients compared with the healthy group. It was also observed that CI and IS were closely associated with internal glucose metabolism. The intestinal gut disturbance of CI patients may be one of the causes inducing CI by glucose metabolism and maybe considered as a potential method to predict the disease.
\end{abstract}

\section{Introduction}

Cerebral infarction (CI) and ischemia (IS) are common diseases in elderly people seriously affecting the quality of

Correspondence to: Dr Yu Zhu or Professor Ping Yang, Department of Clinical Laboratory, Tianjin Huanhu Hospital, Tianjin Key Laboratory of Cerebral Vessels and Neural Degeneration, 6 Jizhao Road, Tianjin 300350, P.R. China

E-mail: zhuyutj@126.com

E-mail: tjhhhospitallab@126.com

*Contributed equally

Key words: cerebral infarction, intestinal microbial communities, sequencing technology life. Patients with IS exhibit insufficient blood supply, which is considered an early stage of CI $(1,2)$. It is predicted that by 2030 , the Chinese population aged $\geq 60$ will be $>300$ million, and $\sim 2 / 3$ patients with primary cerebral vascular disease will be aged $\geq 60$ years. In recent years, due to personal habits or environmental factors, the age of patients with primary cerebral vascular disease has gradually reduced $(3,4)$. Cerebrovascular disease and CI are currently the leading cause of disability and the second leading cause of mortality in China $(5,6)$. Patients with cerebrovascular disease who survive are prone to suffer from the disease again or more than once, $\sim 3 / 4$ of them lost their ability to work to varying extents and among them $40 \%$ are severely disabled. Expensive treatments for cerebrovascular disease have meant great mental and financial burdens on many patients and families. Although in recent years the treatment level of CI has been improved, treatment of the majority of patients remains unsatisfactory, with high mortality and disability rates and poor prognosis. Therefore, investigating the pathogenesis of CI and preventing it is considered an economical and efficient way to control the disease.

The intestinal microbial ecosystem has been widely studied in the past several years (7). The present study hypothesizes that diet can affect the intestinal gut and that variations in intestinal microbial communities can also affect human glucose and lipid metabolism; and variations in glucose and lipid metabolism have already been suggested to be potential causes of CI (8). Therefore, the present study adopted high throughput sequencing technology, which is the latest method to analyze the microbial communities of the intestinal gut, to investigate the intestinal guts of healthy volunteers, CI patients and IS patients, to explore the relation between CI, IS and the intestinal gut, and to consider the utility of using the intestinal gut as a diagnostic marker of CI and IS.

\section{Materials and methods}

Patients. A total of 10 patients were selected; 8 with CI and 2 with IS. They were recruited from Tianjin Huanhu Hospital (Tianjin, China) between May 2015 and October 2015, and had 
A

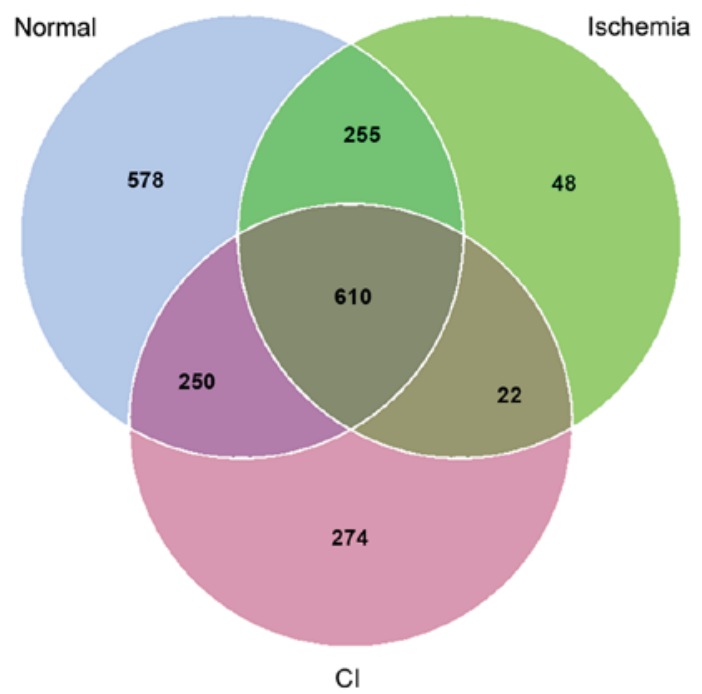

\section{B}
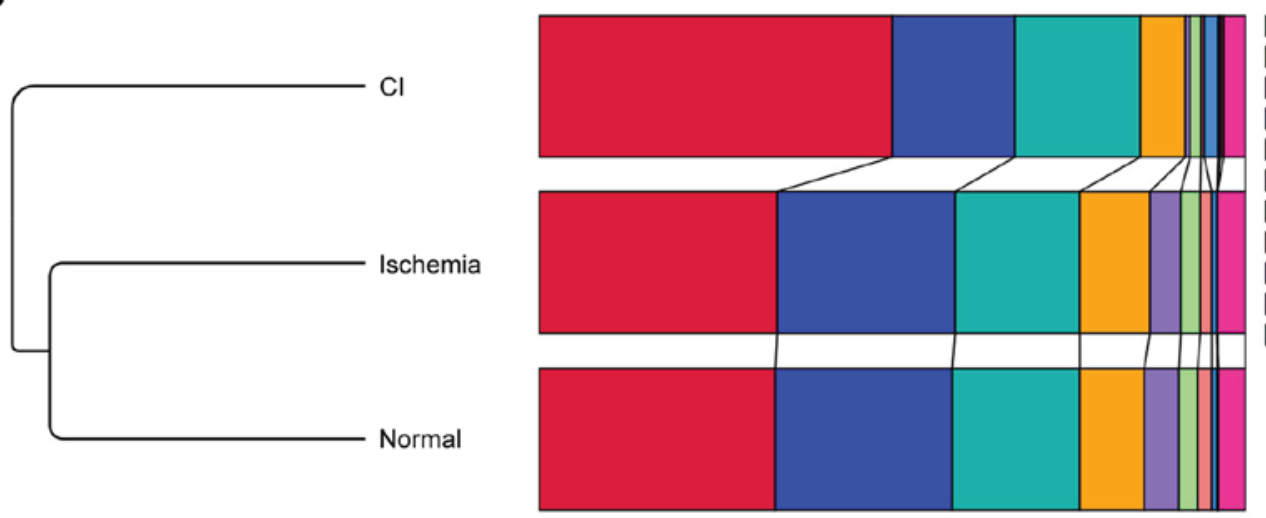

Firmicutes

$\square$ Proteobacteria

$\square$ Bacteroidetes

$\square$ Actinobacteria

$\square$ Cyanobacteria

$\square$ Verrucomicrobia

$\square$ Planctomycetes

$\square$ Tenericutes

$\square$ Euryarchaeota

$\square$ Spirochaetes

Others

Unweighted unifrac distance

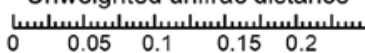

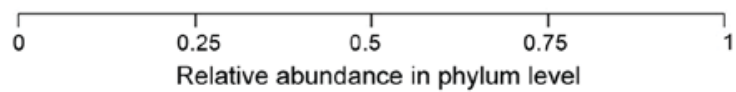

Figure 1. Analysis of the variation of intestinal gut at the phylum level in healthy subjects and patients with CI and IS. (A) The key operational taxonomic units associated with healthy subjects and patients with CI and IS. (B) The UPGMA analysis of the intestinal gut of healthy subjects and patients with CI and IS. CI, cerebral infarction; IS, ischemia; UPGMA, unweighted pair group method with arithmetic mean.

been diagnosed by skull computed tomography examination. The group comprised 6 males and 4 females. Additionally, 10 healthy volunteers, 5 male and 5 female, were selected as the normal group. The age of the individuals was from 53-82 years with a mean age of 64.3. Research subjects did not receive antibiotics in the week prior to the specimen collection. As required by the medical ethics committee, those in the normal group were examined to ensure that they had no metabolic, cardiovascular or cerebrovascular diseases or cancer. All participants signed informed consent prior to the experiment. The study was approved by the Ethics Committee of Tianjin Huanhu Hospital.

Intestinal gut DNA extraction. A $300 \mathrm{mg}$ middle segment feces sample was collected in the morning and the genomic DNA was extracted using the Stool DNA kit (Tiangen Biotech Co., Ltd., Beijing, China). Bacteria cells were split at $95^{\circ} \mathrm{C}$ for 10 min and $4 \mu \mathrm{l}$ RNase A was added and incubated at $70^{\circ} \mathrm{C}$ for $30 \mathrm{~min}$. DNA concentrations were determined using a microplate reader (Multiskan FC, Thermo Fisher Scientific, Inc., Waltham, MA, USA) and stored at $-80^{\circ} \mathrm{C}$.
Polymerase chain reaction (PCR) amplification and Illumina sequencing. The bacterial genomic DNA was amplified with primers specific for the V4 hypervariable regions of the 16SrDNA gene. The sequences were forward, 5'-GTGCCA GCMGCCGCGGTAA-3' and reverse, 5'-GGACTACHV GGGTWTCTAAT-3', and the reaction was performed using Phusion High-Fidelity PCR master mix with GC buffer (New England Biolabs, Inc., Ipswich, MA, USA). Samples were sequenced by Illumina MiSeq platform provided by Illumina, Inc. (San Diego, CA, USA) with the following conditions: Denaturation $1 \mathrm{~min}$ at $98^{\circ} \mathrm{C}, 10 \mathrm{sec}$ at $98^{\circ} \mathrm{C}, 30 \mathrm{sec}$ at $50^{\circ} \mathrm{C}$ and $30 \mathrm{sec}$ at $72^{\circ} \mathrm{C}$ for 30 cycles, and $72^{\circ} \mathrm{C}$ at $5 \mathrm{~min}$.

Serum glucose and lipid detection. A total of $5 \mathrm{ml}$ venous blood was collected from the elbow of the subjects, early in the morning and before they had eaten, and placed in a coagulation-promoting tube then centrifuged at $300 \mathrm{x} \mathrm{g}$ for $10 \mathrm{~min}$ at room temperature. The serum was collected and the blood glucose and lipid concentrations ascertained using a Beckman AU5800 biochemical detector (Beckman Coulter, Inc., Brea, CA, USA). 
A

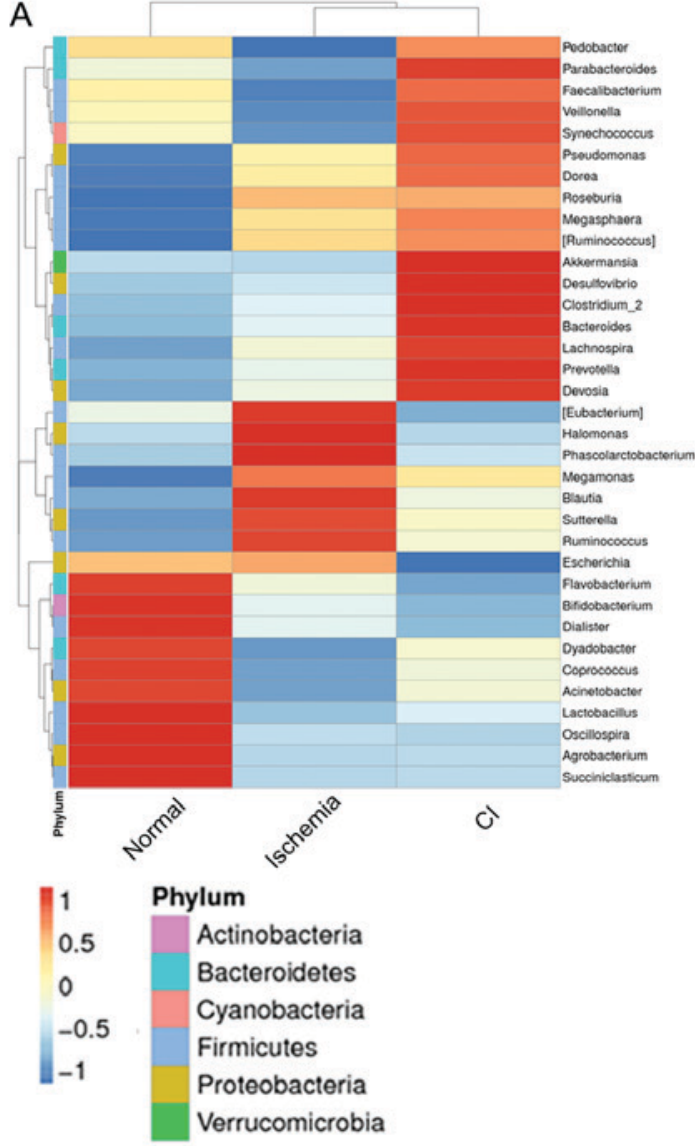

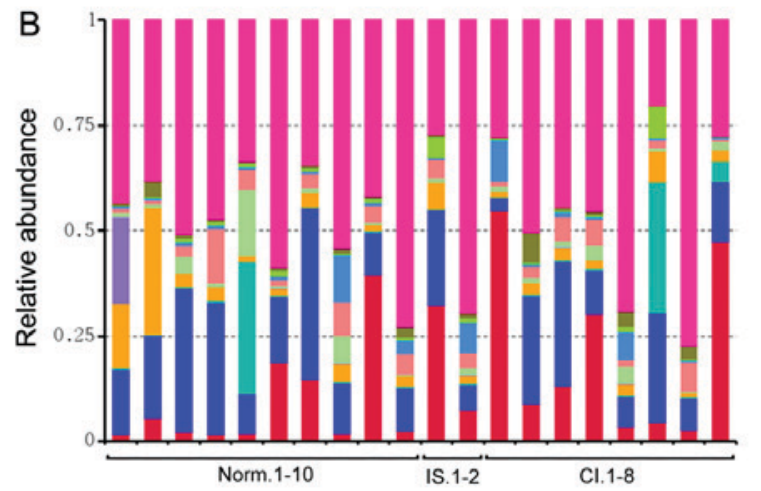

Others
Ruminococcus
Bifidobacterium

Bifidobacte

- Faecalibacterium

-1] Prevotella

- Akkermansia

- Parabacterodes

- Megamonas

Bacteroides

Escherichia

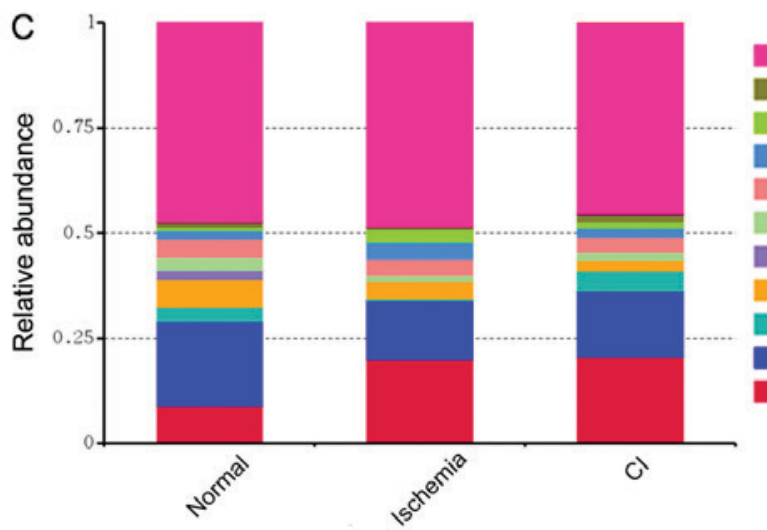

Others

Ruminococcus

Bifidobacterium

Dialister

Faecalibacterium

Prevotella

Akkermansia

Parabacterodes

Megamonas

Bacteroides

Escherichia

Figure 2. Analysis of the variation of intestinal gut in genus level in healthy subjects and CI and IS patients. (A) The heatmap of microorganisms in the intestinal gut at the genus level in healthy subjects and CI and IS patients. Red signifies an increased level and blue signifies a decreased level. (B) The relative abundance of each genus in the intestinal gut in each sample from healthy subjects, and CI and IS patients. (C) The relative abundance of each genus in intestinal gut samples from the healthy subjects $(n=10)$ and patients with $\mathrm{CI}(\mathrm{n}=8)$ and IS $(\mathrm{n}=2)$. CI, cerebral infarction; IS, ischemia.

Statistical analysis. Sequencing results analysis was performed by the QIIME software package 1.7.0 (qiime.org) (9) and UPARSE pipeline (10). Weighted/unweighted UniFrac analysis was performed by QIIME. Cluster samples analysis was performed by principal coordinate analysis (PCoA) and unweighted pair group method with arithmetic mean (UPGMA). Operational taxonomic units (OTUs) analysis was performed by partial least square discriminate analysis via Simca-p+11.5, values higher than 1.5 were considered as key OTUs. The heatmap was constructed with HemI version 1.0 (The CUCKOO Workgroup, Wuhan, Hubei, China). Data analysis was performed using SPSS 11.0 software (SPSS, Inc., Chicago, IL, USA) and one-way analysis of variance with Tukey's test was applied for the intergroup analysis. Values are presented as the mean \pm standard deviation. $\mathrm{P}<0.05$ was considered to indicate a statistically significant difference.

\section{Results}

Microbiota of intestinal gut in CI and IS patients. The sequencing results demonstrated that there were 274 (48key) OTUs that were significantly associated with CI and IS (Fig. 1A). A significant difference in the abundance of Firmicutes, Proteobacteria, Bacteroidetes, Actinobacteria, Cyanobacteria, Verrucomicrobia, Planctomycetes, Tenericutes, Euryarchaeota and Spirochaetesat the phylum level in the CI group compared with the normal group was observed using UPGMA analysis $(\mathrm{P}<0.05)$, however, no significant difference was observed in the abundance of the above phyla in the IS group compared with the normal group (Fig. 1B).

The heatmap demonstrates that there was a change in abundance at the genus level among the groups (Fig. 2A). Particularly, the results demonstrated that Escherichia, Megamonas, Dialister, Bifidobacterium and Ruminococcus were more abundant in the IS and CI groups. The abundance of Bacteroides, Parabacteroides, Akkermansia, Prevotella and Faecalibacterium was decreased in the CI group compared with the normal group $(\mathrm{P}<0.05)$. There was a greater abundance of Escherichia, Dialister and Bifidobacterium in the IS group compared with the normal group $(\mathrm{P}<0.05)$, although the abundance of Bacteroides, Megamonas, Parabacteroides, Akkermansia, Prevotella, Faecalibacterium and Ruminococcus was less. There was a greater abundance of Escherichia, Bacteroides, Megamonas, Prevotella and Ruminococcusin the CI compared with the IS group; although the abundance of Parabacteroides, Akkermansia, Faecalibacterium, Dialister and Bifidobacterium was less $(\mathrm{P}<0.05$; Fig. 2B and $\mathrm{C})$.

Diversities between CI and IS patients' intestinal gut. It can be seen from the principal component analysis (PCA) that the diversities were assessed by a net separation of the three 
A

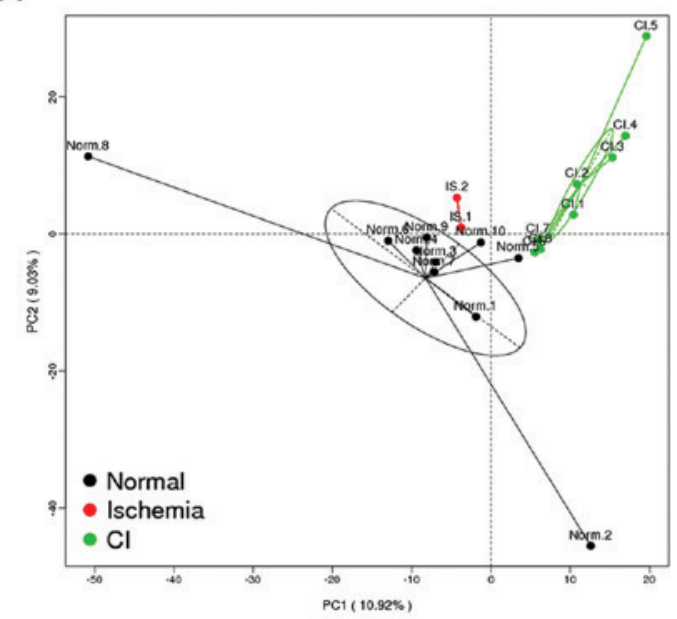

B

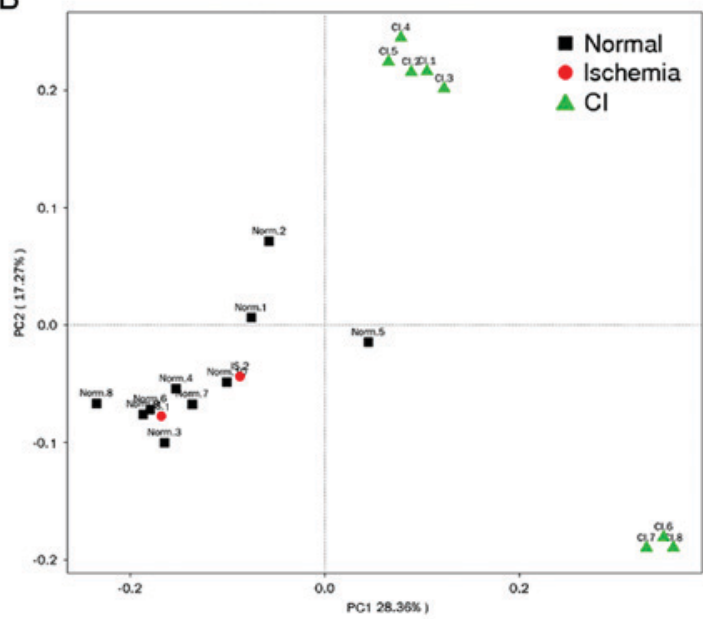

Figure 3. Differences in intestinal gut among healthy subjects and CI and IS patients. (A) Principal component analysis of intestinal gut in healthy subjects, and patients with CI and IS. (B) Principal coordinate analysis of intestinal gut in healthy subjects, and patients with CI and IS. CI, cerebral infarction; IS, ischemia.

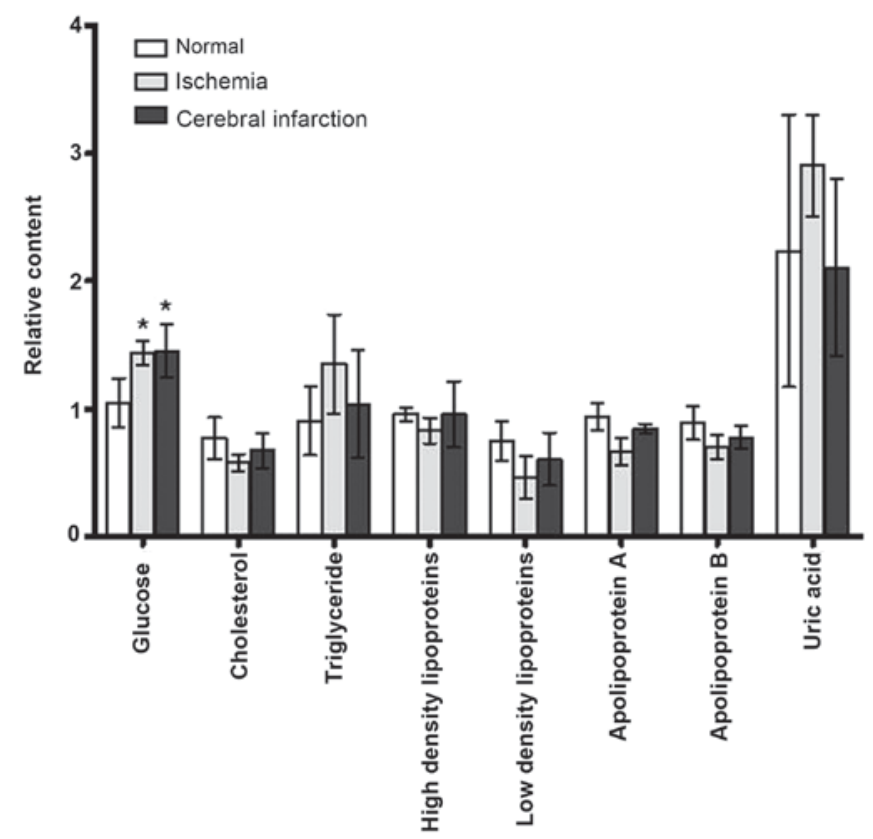

Figure 4. Serum glucose level and lipid metabolism of healthy subjects, and patients with cerebral infarction and ischemia. The graph presents the level of glucose, triglyceride, total cholesterol, high-density lipoprotein and low-density lipoprotein, apolipoprotein A, apolipoprotein B and uric acid. Values are presented as the mean \pm standard deviation. ${ }^{*} \mathrm{P}<0.05$ vs. healthy group.

groups produced by the intestinal gut between the healthy group and the CI and IS patients $(\mathrm{P}<0.05)$, which indicated less similarity in the microbial structure and composition of the feces between CI and IS patients and the healthy group (Fig. 3A). Notably, the same pattern was observed in the PCoA analysis and that there are also samples that were grouped into two distinct clusters in the intestinal microbial communities of CI patients $(\mathrm{P}<0.05)$; this may be associated with the process of $\mathrm{CI}$ and requires further investigation (Fig. 3B).

Level of serum glucose and lipid metabolism in patients with CI and IS, and the healthy group. The present study demonstrated that the blood glucose of patients with CI and
IS was significantly higher compared with the healthy group; however, no significant changes were observed in triglyceride, total cholesterol, high-density lipoprotein and low-density lipoprotein, apolipoprotein A, apolipoprotein B and uric acid ( $\mathrm{P}<0.05$; Fig. 4). Therefore, it is hypothesized that variations in the intestinal microbial communities in CI and IS patients may lead to a disorder of the blood glucose metabolism and thus a potential dangerous factor causing CI.

\section{Discussion}

CI refers to focal cerebral ischemic necrosis or encephalomalacia caused by the disturbance of the cerebral circulation, IS and hypoxia. It has high morbidity, disability and mortality, and seriously endangers human life (11). Therefore, exploring its mechanism with the aim of predicting and preventing CI has become an important area of study.

The present study demonstrated that the intestinal gut serves an important function in cardiovascular and cerebrovascular diseases and a previous study considered its role in cardiovascular disease (12). The intestinal gut can affect the intestinal permeability through glucose metabolism, increase the quantity of harmful substances in the blood and stimulate the inflammatory response, thus promoting the occurrence and development of the diseases $(13,14)$. However, at present little is known about the variations in the intestinal gut of patients with cerebral arterial thrombosis. The development of the new-generation high throughput sequencing technology offers the opportunity to rapidly acquire and analyze the information on the intestinal microbial community composition, function discovery, metabolic function and other aspects, due to its digitized signal, high data flux, deep sequencing depth, high accuracy and other features, leading to an improved understanding of the intestinal gut.

Glycolysis breaks down glucose and is essential for human biological function. Escherichia are considered to serve an important function in the mechanism of glycolytic pathways for regulating glucose catabolism (15). Previous studies have demonstrated that decreased relative abundance of 
Akkermansia and increased relative abundance of Bacteroides, Ruminococcus and Parabacteroides in the gut microbiome can improve glucose homeostasis in obese mice and provide a catabolic pathway through a novel mannosylglucose phosphorylase, suggesting that Akkermansia, Bacteroides, Ruminococcus and Parabacteroides may serve an important function in the mechanism of glycometabolism (16-19).There appears to be a significant enrichment of Megamonas in Chinese people compared with other ethnicities, and it may serve a potential glycometabolism role (20). Prevotella has been demonstrated to serve a role in glycometabolism and in promoting increased glycogen storage (21). The abundance of Faecalibacterium and Dialister, and the abundance of Bifidobacterium in patients with type 2 diabetes, was increased by altering lipid and glucose metabolism, glucose tolerance and the inflammatory immune responses (22-25).

By exploring the intestinal microbial communities of patients with CI, the present study identified that the abundance of Escherichia, Bacteroides, Megamonas, Parabacteroides, Akkermansia, Prevotella, Faecalibacterium, Dialister, Bifidobacterium and Ruminococcus in intestinal microbial communities was significantly different inpatients with CI and IS compared with healthy subjects, and there were also significant differences in the intestinal microbial profile among different patients with CI by PCA and PCoA analysis; this may be associated with the process of $\mathrm{CI}$ and may provide a new basis for diagnosing and classifying CI patients and understanding CI pathogenesis.

In conclusion, we hypothesized that microbial genus profile may be markedly changed prior to the onset of CI and IS and searching for the characteristic intestinal gut of CI patients may provide a new basis for the diagnosis and a possible indicator for prevention and provide a major social and economic benefit. However, there were also certain limitations of the present study, for example, the patient samples sizes for CI and IS were small, and a study on a much larger number will be required to validate these results.

\section{Acknowledgements}

The present study was supported by National Natural Science Foundation of China (grant no. 31501159), Tianjin Public Health Key Research Project (grant no. 15KG108), the Tianjin Research Program of Application Foundation and Advanced Technology (grant no. 14JCQNJC12000), Tianjin Science and Technology Key Project on Chronic Diseases Prevention and Treatment (grant no. 16ZXMJSY00020) and Special Program of Talents Development for Excellent Youth Scholars in Tianjin, China.

\section{References}

1. Zhang T, Wang W, Huang J, Liu X, Zhang $\mathrm{H}$ and Zhang N: Metabolomic investigation of regional brain tissue dysfunctions induced by global cerebral ischemia. BMC Neurosci 17: 25, 2016.

2. Mitha AP, Wong JH, Hill MD and Goyal M: Introducing a new era of ischemic stroke care. J Neurosurg 125: 508-511, 2016.

3. Zuo X, Hou Q, Jin J, Zhan L, Li X, Sun W, Lin K and Xu E: Inhibition of cathepsin B alleviates secondary degeneration in ipsilateral thalamus after focal cerebral infarction in adult rats. J Neuropathol Exp Neuro 75: 816-826, 2016.

4. Shen B, Liu Q, Gu Y, Wang Y and Zhang Z: Efficacy and safety evaluation on arterial thrombolysis in treating acute cerebral infarction. Cell Biochem Biophys 73: 297-304, 2015.
5. Sun Y,He W and Geng L: Neuroprotective mechanism of HIF-1a overexpression in the early stage of acute cerebral infarction in rats. Exp Ther Med 12: 391-395, 2016.

6. Ma XM, Liu M, Liu YY, Ma LL, Jiang Y and Chen XH: Ischemic preconditioning protects against ischemic brain injury. Neural Regen Res 11: 765-770, 2016.

7. Fu J,Bonder MJ,Cenit MC, TigchelaarEF, Maatman A, Dekens JA, Brandsma E, Marczynska J, Imhann F, Weersma RK, et al: The Gut microbiome contributes to a Substantial Proportion of the variation in blood lipids. Circ Res 117: 817-824, 2015.

8. Yin J, Liao SX, He Y, Wang S, Xia GH, Liu FT, Zhu JJ, You C, Chen Q, Zhou L, et al: Dysbiosis of gut microbiota with reduced trimethylamine-n-oxide level in patients with large-artery atherosclerotic stroke or transient ischemic attack. J Am Heart Assoc 4: e002699, 2015.

9. Caporaso JG, Kuczynski J, Stombaugh J, Bittinger K, Bushman FD, Costello EK, Fierer N, Peña AG, Goodrich JK, Gordon JI, et al: QIIME allows analysis of high-throughput community sequencing data. Nat Methods 7: 335-336, 2010.

10. Edgar RC: UPARSE: Highly accurate OTU sequences from microbial amplicon reads. Nat Methods 10: 996-998, 2013.

11. Yamashita T, Kasahara K, Emoto T, Matsumoto T, Mizoguchi T, Kitano N, Sasaki N and Hirata K: Intestinal immunity and gut microbiota as therapeutic targets for preventing atherosclerotic cardiovascular diseases. Circ J 79: 1882-1890, 2015.

12. Li D, Kirsop J and Tang WH: Listening to Our Gut: Contribution of Gut Microbiota and Cardiovascular Risk in Diabetes Pathogenesis. Curr Diab Rep 15: 63, 2015.

13. Koutsos A, Tuohy KM and Lovegrove JA: Apples and cardiovascular health-is the gut microbiota a core consideration? Nutrients 7: 3959-3998, 2015.

14. Kelly TN, Bazzano LA, Ajami NJ, He H, Zhao J, Petrosino JF, Correa A and He J: Gut microbiome associates with lifetime cardiovascular disease risk profile among bogalusa heart study participants. Circ Res 119: 956-964, 2016.

15. Hollinshead WD, Rodriguez S, Martin HG, Wang G, Baidoo EE, Sale KL, Keasling JD, Mukhopadhyay A and Tang YJ: Examining Escherichia coli glycolytic pathways, catabolite repression, and metabolite channeling using $\Delta \mathrm{pfk}$ mutants. Biotechnol Biofuels 9: 212, 2016.

16. Sung MM, Kim TT, Denou E, Soltys CM, Hamza SM, Byrne NJ, Masson G, Park H, Wishart DS, Madsen KL, et al: Improved glucose homeostasis in obese mice treated with resveratrol is Associated with Alterations in the Gut Microbiome. Diabetes 66: 418-425, 2017.

17. Senoura T, Ito S, Taguchi H, Higa M, Hamada S, Matsui H, Ozawa T, Jin S, Watanabe J, Wasaki J and Ito S: New microbial mannan catabolic pathway that involves a novel mannosylglucose phosphorylase. Biochem Biophys Res Commun 408: 701-706, 2011.

18. Ottman N, Huuskonen L, Reunanen J, Boeren S, Klievink J, Smidt H, Belzer C and de Vos WM: Characterization of outer membrane proteome of Akkermansia muciniphila reveals sets of novel proteins exposed to the human intestine. Front Microbiol 7: 1157, 2016.

19. Tsai YC, Wang HT, Hsu JT, Li YH and Chen CY: Yeast with bacteriocin from ruminal bacteria enhances glucose utilization, reduces ectopic fat accumulation, and alters cecal microbiota in dietary-induced obese mice. Food Funct 6: 2727-2735, 2015.

20. Kuang YS, Li SH, Guo Y, Lu JH, He JR, Luo BJ, Jiang FJ, Shen H, Papasian CJ, Pang H, et al: Composition of gut microbiota in infants in China and global comparison. Sci Rep 6: 36666, 2016.

21. Kovatcheva-Datchary P, Nilsson A, Akrami R, Lee YS, De Vadder F, Arora T, Hallen A, Martens E, Björck I and Bäckhed F: Dietary fiber-induced improvement in glucose metabolism is associated with increased abundance of prevotella. Cell Metab 22: 971-982, 2015.

22. Remely M, Hippe B, Zanner J, Aumueller E, Brath H and Haslberger AG: Gut microbiota of obese, type 2 diabetic individuals is enriched in Faecalibacterium prausnitzii, Akkermansia muciniphila and Peptostreptococcus anaerobius after weight loss. Endocr Metab Immune Disord Drug TargetsAug, 2016 (Epub ahead of print).

23. Ciubotaru I, Green SJ, Kukreja S and Barengolts E: Significant differences in fecal microbiota are associated with various stages of glucose tolerance in African American male veterans. Transl Res 166: 401-411, 2015.

24. Martínez I, Lattimer JM, Hubach KL, Case JA, Yang J, Weber CG, Louk JA, Rose DJ, Kyureghian G, Peterson DA, et al: Gut microbiome composition is linked to whole grain-induced immunological improvements. ISME J 7: 269-280, 2013.

25. Fritsch C, Jänsch A, Ehrmann MA, Toelstede S and Vogel RF: Characterization of cinnamoyl esterases from different lactobacilli and bifidobacteria. Curr Microbiol 74: 247-256, 2017. 